\title{
Mapping genes in barley for resistance to Puccinia coronata from couch grass and to $P$. striiformis from brome, wheat and barley
}

\author{
Rients E. Niks $\cdot$ Sisay K. Alemu • \\ Thierry C. Marcel $\cdot$ Skye van Heyzen
}

Received: 13 March 2015/Accepted: 6 July 2015/Published online: 22 July 2015

(C) The Author(s) 2015. This article is published with open access at Springerlink.com

\begin{abstract}
Barley (Hordeum vulgare L.) mapping populations have been developed that are useful to study the inheritance of quantitative resistance to adapted and unadapted rust fungi. In a recent host range study, we found that the parents of those mapping populations also differed in their resistance to the crown rust Puccinia coronata (PcE) of couch grass (Elymus repens), as well as three isolates of $P$. striiformis, representing formae speciales hordei (Psh), tritici (Pst) and bromi (Psb). Available mapping populations were phenotyped at the seedling stage to map the genes conferring resistance to these rust
\end{abstract}

Electronic supplementary material The online version of this article (doi:10.1007/s10681-015-1516-y) contains supplementary material, which is available to authorized users.

R. E. Niks $(\bowtie)$

Laboratory of Plant Breeding, Graduate School for

Experimental Plant Sciences, Wageningen University,

Droevendaalsesteeg 1, 6708 PB Wageningen,

The Netherlands

e-mail: rients.niks@wur.nl

Present Address:

S. K. Alemu

Agricultural Biotechnology Research Laboratory, Holetta

Research Center, Ethiopian Institute of Agricultural

Research, Holetta, Ethiopia

Present Address:

T. C. Marcel

INRA, UMR1290, BIOGER, 78850 Thiverval-Grignon,

France isolates. Resistances to $P c E, P s b$ and Pst inherit quantitatively. This contrasted with reports that barley nonhost resistance to unadapted formae speciales of $P$. striiformis is based on major genes. We mapped QTLs effective against $P c E$ using relative latency period and relative infection frequency. Some QTLs for resistance were contributed by 'Vada' and 'Cebada Capa', others by SusPtrit. One PcE-resistance QTL on $3 \mathrm{H}$, contributed by 'Cebada Capa', co-localised with a QTL effective against four unadapted grass rust species, indicating either a single gene with broad-spectrum effectiveness or a cluster of rather specific genes. Chromosome arm 7HL from Vada seems particularly rich in genes for resistance to rust fungi. Resistance to $P s t$ and $P s b$, measured as the number of uredinia, tended to co-localise with each other and mapped to

\author{
T. C. Marcel \\ AgroParisTech, UMR1290, BIOGER, \\ 78850 Thiverval-Grignon, France \\ Present Address: \\ S. van Heyzen \\ KWS-UK Ltd., Thriplow, Royston, \\ Hertfordshire SG8 7RE, UK
}


1HS, 2HL and 7HL. The nearly complete resistance of Ethiopian line L94 to Psh was due to a major gene mapped on chromosome $4 \mathrm{H}$. That gene is likely the recessive gene $\operatorname{rps} G Z$, which was previously reported in the Ethiopian line Grannenlose Zweizeilige.

Keywords QTLs · Nonhost resistance - Barley · Heterologous and adapted rusts $\cdot$ Stripe rust $\cdot$ Crown rust

\section{Introduction}

Resistance genes are used by plant breeders to develop new cultivars that are more resistant to economically important diseases. Most economically relevant pathogens are specialised to infect only one or a few plant species, with other plants species being classified as nonhosts. Host resistance genes can either confer a quantitatively inherited reduction of susceptibility (socalled minor genes) or a qualitatively inherited hypersensitive resistance (so-called major $R$-genes) (Niks 1987). Within host resistance, partial and hypersensitive resistances are thought to be controlled by different sets of genes and to differ in their durability (Niks and Marcel 2009). Partial resistance is defined as a quantitative resistance that is not based on a hypersensitive reaction. The inheritance of nonhost resistance has only begun to be thoroughly investigated and may be based either on Pathogen-associated molecular pattern (PAMP)-triggered immunity (PTI) or on effective $R$-genes in the plant corresponding to cognate effectors in the unadapted pathogen (SchulzeLefert and Panstruga 2011).

In recent decades, barley (Hordeum vulgare L.) has proven not only to be an excellent model species to investigate the genetic basis of both types of host resistance (Niks et al. 2000; Collins et al. 2007) but also to study the inheritance of nonhost resistance to the specialised biotroph pathogenic fungi Blumeria graminis and Puccinia species (Atienza et al. 2004; Aghnoum et al. 2010; Aghnoum and Niks 2010; Niks 2014). The suitability of barley for studies on the inheritance of nonhost resistance is due to its 'nearnonhost' status to heterologous (i.e., unadapted) Puccinia rust species and Blumeria graminis formae speciales. In particular, the experimental research line SusPtrit (Atienza et al. 2004), which has been developed to have extraordinarily high susceptibility to heterologous rust fungi, proved to be instrumental in elucidating the inheritance of nonhost resistance. Mapping populations were developed with SusPtrit using the regular, immune, cvs Vada, Cebada Capa and Golden Promise as resistant parents (Jafary et al. 2006, 2008; Yeo et al. 2014).

Crown rust, a fungal disease of small-grain cereals and grass species across the world, is caused by the Puccinia coronata species complex. This disease is particularly common and damaging in ryegrass (Lolium) and oats (Avena) but is one of the less frequently reported rust fungal pathogens of cultivated barley (H. vulgare) and wheat (Triticum) (Niks et al. 2013). A screen of 108 barley accessions (Niks et al. 2013) with a recently collected European isolate of $P$. coronata on Elymus repens (PCE) confirmed that susceptibility is the rule in barley at the seedling stage (Jin and Steffenson 2002). None of the accessions had complete resistance, and only approximately $17 \%$ of the accessions showed substantially reduced uredinium development. Among the quantitatively resistant accessions were cvs Vada and Cebada Capa, whereas line SusPtrit was among the most susceptible accessions (Niks et al. 2013).

Previously, the host status of barley was quantified for three isolates of $P$. striiformis, belonging to three formae speciales: f.sp. tritici (Pst), f.sp. bromi (Pst), and f.sp. hordei (Psh) (Niks et al. 2013). Pst could infect only a small minority (10\%) of the accessions. $P s b$ was somewhat more adapted to barley (47\%), and the barley-adapted $P$ sh could infect the vast majority of the barley accessions (90\%) (Niks et al. 2013). As we found previously (Atienza et al. 2004) for other unadapted rust fungi, cvs Vada and Cebada Capa were also immune to Pst and $P s b$ and contrasted with the highly susceptible SusPtrit. Line L94 was highly resistant to $P s h$, to which cv Vada was susceptible.

The availability of mapping populations Cebada Capa/SusPtrit, Vada/SusPtrit and L94/Vada allowed us to determine the genes responsible for resistance to $P c E, P s t, P s b$ and Psh. The aim of our study was to determine whether resistances to these four rust fungi is inherited in a monogenic or polygenic manner and whether the resistance genes are rust fungus specific and cultivar specific. We also set out to determine whether identified resistance genes map to chromosome regions that were previously reported to confer resistance in the same mapping populations to various 
species of homologous and heterologous rust fungi (Jafary et al. 2006, 2008; Qi et al. 1998; Marcel et al. 2007).

\section{Materials and methods}

Pathogen material

We used the following rust isolates to test the mapping populations: one isolate of the crown rust fungus, Puccinia coronata, collected from couch grass ( $E$. repens) in Hungary (labelled as $P c E$ ) and identified as P. coronata var. hordei (Niks et al. 2013); one isolate of Puccinia striiformis f.sp. bromi (Psb) collected from California brome grass (Bromus carinatus) in Wageningen; isolate 237E141 of Puccinia striiformis f.sp. tritici (Pst); and isolate 24 of $P$. striiformis f.sp. hordei (Psh) (Niks et al. 2013). The latter two isolates were kindly provided by the Global Yellow Rust Gene Bank (Plant Research International, Wageningen, Netherlands), which has recently been transferred to Aarhus University in Denmark. Isolates $P c E$ and $P s b$ were each collected from a single host plant and were not further purified in the greenhouse by mono-spore or monopustule culture.

$P c E$ was propagated on susceptible clones of $E$. repens. The $P$. striiformis isolates were propagated in separate greenhouse compartments on accessions of grasses and cereals that in preliminary trials had proven to be susceptible (Niks et al. 2013). Surplus inoculum was stored in liquid nitrogen until required, but mostly fresh inoculum was used in the experiments.

\section{Plant materials}

Three mapping populations of barley (H. vulgare L.) were used in this experiment (Table 1). These mapping populations were developed at the Laboratory of Plant Breeding of Wageningen University. Resistance to $P c E$ was mapped on $\mathrm{F}_{8}$ to $\mathrm{F}_{9}$-derived recombinant inbred line (RIL) mapping populations Vada/SusPtrit (V/S: 140 RILs: Jafary et al. 2006) and Cebada Capa/ SusPtrit (CC/S: 110 RILs: Jafary et al. 2008). Resistance to $P s b$ and $P s t$ was mapped on $\mathrm{V} / \mathrm{S}$ and resistance to $P s h$ on L94/Vada (L/V: 103 RILs: Qi et al. 1998). Vada is a commercial spring barley line developed at Wageningen, popularly grown in West-
Table 1 Mapping populations used to map genes for resistance to four rust isolates, as well as the resistance level of the parental lines, as determined in Niks et al. (2013)

\begin{tabular}{|c|c|c|c|}
\hline \multirow[t]{3}{*}{ Parental line } & \multicolumn{3}{|c|}{ Rust isolates and mapping populations ${ }^{\mathrm{a}}$} \\
\hline & \multirow{2}{*}{$\begin{array}{l}\text { P. coronata } \\
P c E \\
\mathrm{CC} / \mathrm{S} \text { and } \mathrm{V} / \mathrm{S}\end{array}$} & \multicolumn{2}{|l|}{ P. striiformis } \\
\hline & & $\begin{array}{l}P s b \text { and } P s t \\
\text { V/S }\end{array}$ & $\begin{array}{l}\text { Psh } \\
\mathrm{L} / \mathrm{V}\end{array}$ \\
\hline SusPtrit (S) & $\mathrm{S}$ & $\mathrm{S}$ & \\
\hline Cebada Capa (CC) & PR & & \\
\hline Vada (V) & PR & I & $\mathrm{S}$ \\
\hline L94 (L) & & & $\mathrm{R}$ \\
\hline
\end{tabular}

$S$ susceptible, $P R$ partially resistant, $R$ highly resistant, $I$ immune

a PcE: Puccinia coronata collected from Elymus repens; Psb: P. striiformis collected from Bromus carinatus; Pst: P. striiformis f.sp. tritici; Psh: P. striiformis f.sp. hordei

Europe in the late 1950s and in the 1960s. Cebada Capa is an Argentinean spring barley from before 1940. L94 is a spring barley line drawn from an Ethiopian land race and is also known as Abyssinian 1102, HOR3036 and BBA1465 (see Jørgensen 1992). These parental lines had partial or strong resistance to the four rust isolates (Table 1). The parental line SusPtrit is an experimental line in which genes for susceptibility to $P$. triticina were accumulated (Atienza et al. 2004). This line is also susceptible at the seedling stage to several other heterologous rust fungi, including Psb and Pst, to which Cebada Capa and Vada were immune (Niks et al. 2013). SusPtrit is very susceptible to $P c E$.

Inoculation and incubation of the mapping populations

Seedlings were grown in plant boxes. Each box contained the two parental lines as references. Several boxes were required to accommodate the whole mapping population. Each isolate was tested in two to three consecutive experiments (see below). Plant boxes were kept in a greenhouse compartment at $20{ }^{\circ} \mathrm{C} / 16 \mathrm{~h}$ day and $16{ }^{\circ} \mathrm{C} / 8 \mathrm{~h}$ night; at daytime, supplementary light was provided when light intensities were lower than $150 \mathrm{Watt} / \mathrm{m}^{2}$. Relative humidity was kept at approximately $70 \%$.

Eleven days after sowing, the first seedling leaves were fixed in a horizontal position by iron pins, with the adaxial surface facing upwards for inoculation. 
Inoculation took place in a settling tower or by midpoint inoculation (see below). After inoculation, the plant boxes were transferred to a dark dew chamber overnight, at $16{ }^{\circ} \mathrm{C}$. The next morning, the iron pins were removed so that seedlings could assume their upright position again. The boxes were transferred to another greenhouse compartment with identical conditions as the seedling growth compartment.

\section{$P c E$}

Each RIL was represented by a single plant, with 32 RILs and one seedling of each parent grown together in each $37 \mathrm{~cm} \times 39 \mathrm{~cm}$ plant box. Inoculation was carried out in a settling tower (Niks et al. 2011) by applying $5 \mathrm{mg}$ of urediniospores per box (300 urediniospores per $\mathrm{cm}^{2}$ ) in the first two experiments of V/S and $3 \mathrm{mg}$ per box $\left(180\right.$ spores per $\left.\mathrm{cm}^{2}\right)$ in the third experiment of $\mathrm{V} / \mathrm{S}$ and for all three experiments of $\mathrm{CC} /$ $\mathrm{S}$. The spores were mixed with approximately $\times 10$ as much inert Lycopodium spores to achieve a homogeneous distribution of the inoculum.

\section{P. striiformis isolates}

Each RIL was represented by three seedlings per $38 \mathrm{~cm} \times 58 \mathrm{~cm}$ plant box, together with parents and SusPtrit. For Pst, wheat cultivar Michigan Amber was added as a susceptible reference, and SusPtrit served as the susceptible reference for $P s h$ and $P s b$. Successful single infection units of the yellow rust fungus form colonies that develop a branching network of fungal mycelium forming a long linear lesion on which many uredinia develop (Chen et al. 2014). This requires different criteria to measure differences in level of quantitative resistance compared to leaf rust, crown rust and stem rust fungi, where each successful infection unit results initially in only one uredinium. A midpoint inoculation technique was used for one experiment using Psh and for two experiments using $P s b$ and Pst. First, leaves of the seedlings were fixed horizontally, and a mark was placed at about twothirds of the distance towards the tip of the leaf using a black marker pen. Using a fine paint brush, a mixture of urediniospores and Lycopodium spores was painted near the mark across the width of the leaf. A third experiment was performed for isolates $P s b$ and $P s t$ using $37 \mathrm{~cm} \times 39 \mathrm{~cm}$ plant boxes and inoculated in the settling tower. In those experiments, $6 \mathrm{mg}$ of urediniospores, mixed with Lycopodium spores (1:20 $\mathrm{v} / \mathrm{v})$, were applied, resulting in 360 spores per $\mathrm{cm}^{2}$.

Observations on the levels of infection

PcE

Latency period (LP) and Infection Frequency (IF) were measured for each inoculated seedling. Just before the first uredinia were visible, a 1- to 2-cm long segment was marked at the central portion of each leaf. In that segment, mature uredinia were counted daily using a pocket lens $(\times 10)$, until the number did not increase anymore (typically 5 or 6 days). The LP of the pathogen on each seedling was estimated by calculating the number of hours from inoculation to the time at which $50 \%$ of the ultimate number of uredinia were visible (Niks et al. 2011). The final number of uredinia were counted in a $1-\mathrm{cm}^{2}$ frame to determine the IF, which was calculated as the number of uredinia per square centimetre.

Relative LP (RLP) and Relative IF (RIF) were calculated to compensate for possible small differences in LP or IF between boxes. Values were taken as relative by setting the average over all items in the box at $100 \%$. We presume that the batches of 32 RILs represent a random sample of the segregating population, and hence, its average should be largely constant between boxes. For both RLP and RIF, the average of the three experiments was considered to represent the level of resistance of each RIL and the parental lines. The Genstat statistical package (10th edition, version 10.2. 0.175) was used to calculate the correlation between the RLP and RIF and to carry out analysis of variance for both RLP and RIF to test differences among the parental lines.

\section{P. striiformis isolates}

Experiments inoculated by midpoint inoculation were assessed at the time when the reference lines SusPtrit (for $P s b$ and $P s h$ ) or Michigan Amber (for Pst) showed between 50 and 100 mature uredinia. The RILs were assessed for the number of uredinia as well as the cumulative lesion length (measured in millimetres as the cumulative length of all lesions present on a single leaf blade).

The seedlings inoculated in the settling tower (performed for one of the three experiments for the 
isolates of $P s b$ and $P s t$ ) were evaluated by daily counting of all uredinia on the seedling leaf, from the moment sporulation started until the rust had produced at least 50 uredinia, unless uredinium formation had stopped. Uredinium formation was considered to have stopped when the number of uredinia on a seedling did not increase for three consecutive days.

Evaluation was performed using the rate of development of the rust in uredinia per hour and the LP in days required for the first uredinium on the leaf to mature, i.e., turning bright yellow and breaking through the epidermis.

\section{QTL mapping and analysis}

The software Map QTL version 5.0 (Van Ooijen 2004) was used to map QTLs using the various quantitative data. Marker data and linkage maps for all three mapping populations were published previously (see Grain Genes; Qi et al. 1998; Jafary et al. 2006, 2008). QTL mapping started with a round of Interval Mapping (IM). In the regions of the putative QTLs ( $\log$ of the likelihood ratio [LOD] $>3.0$ ), the markers with the highest LOD values (peak markers) were taken as co-factors for running a multiple-QTL mapping program, the MQM method (Jansen and Stam 1994). When LOD values of some markers at other regions reached the significance level, MQM was repeated by adding these new peak markers as cofactors until a stable LOD profile was reached. The restricted-MQM (rMQM) method was used to determine the values of the LOD, the proportion of explained phenotypic variation, and the additive effect. QTL support intervals were defined based on the projection of the LOD-1 and LOD-2 cut-offs of the LOD profile on the linkage map (Van Ooijen 1992). A LOD value of 3.0 was taken as the significance threshold value for declaring a QTL. The detected QTLs were added to the genetic maps of the populations using MapChart 2.2 (Voorrips 2002) to visualise their positions.

To compare QTL positions between linkage maps, the confidence intervals and peak markers were marked on the high-density integrated genetic map posted on the GrainGenes website, "Barley, Integrated, Marcel2009" (http://wheat.pw.usda.gov) (Aghnoum et al. 2010). On the integrated map, QTLs for partial resistance to leaf rust $(P$. hordei) and nonhost resistance to various heterologous rusts are indicated, enabling comparison of QTL positions to different rust species and isolates (Jafary et al. 2008).

\section{Results}

\section{QTLs for RLP and RIF of $P c E$}

In V/S, the RILs showed a transgressive segregation for RLP and RIF to PcE (Fig. 1), indicating that both Vada and SusPtrit contributed quantitative resistance alleles to the progeny. Vada tended to be more susceptible than SusPtrit in this set of experiments, but the differences between Vada and SusPtrit in RLP and RIF were not statistically significant. In $\mathrm{CC} / \mathrm{S}$, the transgression was less conspicuous, with Cebada Capa being the more resistant parent. In both mapping populations, the correlation coefficient between RLP and RIF was -0.55 .

In both populations, we found four QTLs that conferred quantitative resistance to $P c E$, with longer LP or lower IF or both (Table 2; Fig. 2). The QTLs were provisionally named Rpcql to Rpcq 8 (Table 2). In V/S, SusPtrit contributed two significant QTLs for resistance. Rpcq2 was more prominent for RLP, and Rpcq4 was only found for RIF. They both mapped to linkage group $5 \mathrm{H}$ (Supplementary Fig. 1). These QTLs could represent one locus, as the LOD-2 intervals overlap (Fig. 2). In particular, Rpcq4 had a very wide LOD-2 interval. However, due to the large distance between the peak markers, the shape of the LOD profiles (Supplementary Fig. 1) and the fact that Rpcq4 did not appear to affect the RLP, we tentatively present Rpcq 2 and Rpcq4 here as two separate loci. In $\mathrm{CC} / \mathrm{S}$, SusPtrit contributed one QTL for resistance, Rpcq8, located on $7 \mathrm{H}$. This QTL mapped to the telomeric region of the short arm of chromosome $7 \mathrm{H}$, (7HS) at $0 \mathrm{cM}$. The peak marker, as well as eight other markers linked to it, had a skewed segregation. Peak marker E39M48-310 deviated from the expected 1:1 segregation, having 86 RILs carrying the SusPtrit allele and 21 carrying the 'Cebada Capa' allele. In the integrated map, the marker was represented by the nearby marker E38M61-128, with a somewhat less skewed segregation $(75: 25)$. The latter marker was positioned on the integrated map (Aghnoum et al. 2010) at position $48.7 \mathrm{cM}$. This discrepancy between the linkage map CC/S and the integrated map suggests that the telomeric region of $7 \mathrm{HS}$ in $\mathrm{CC} / \mathrm{S}$ has some 


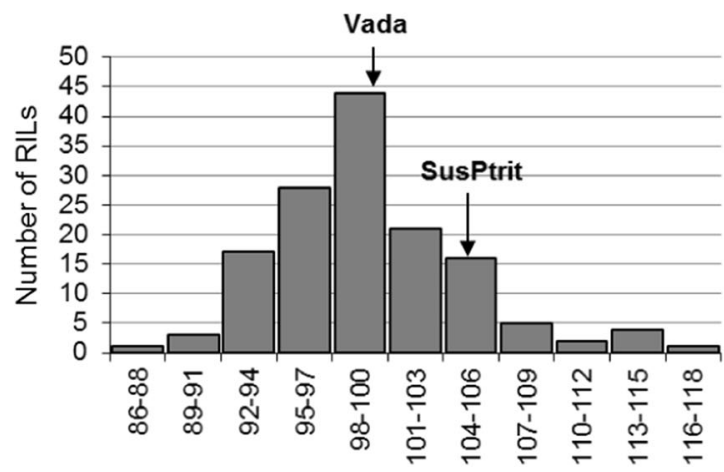

Relative Latency Period (RLP)

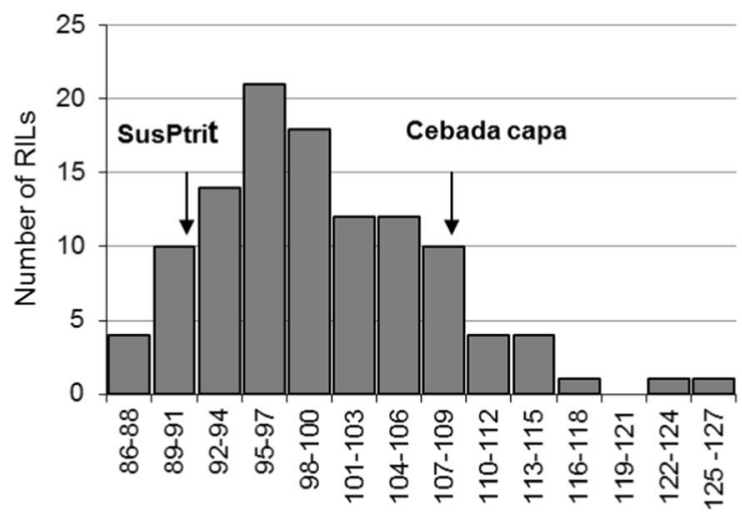

Relative Latency Period (RLP)

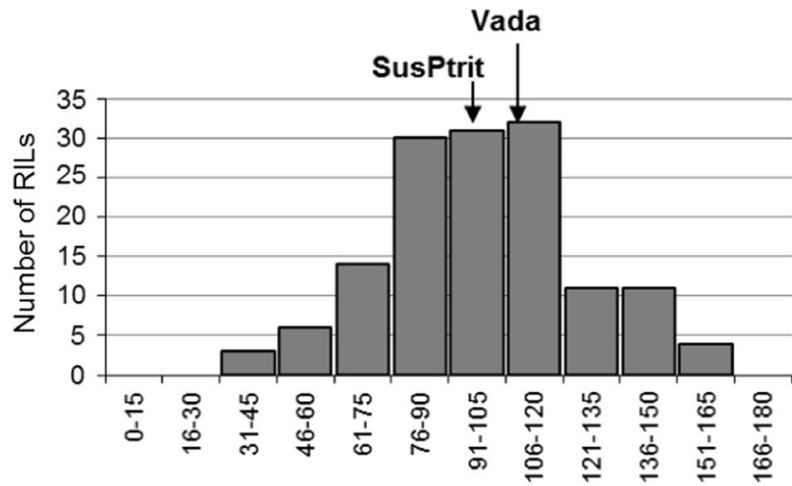

Relative Infection Frequency (RIF)

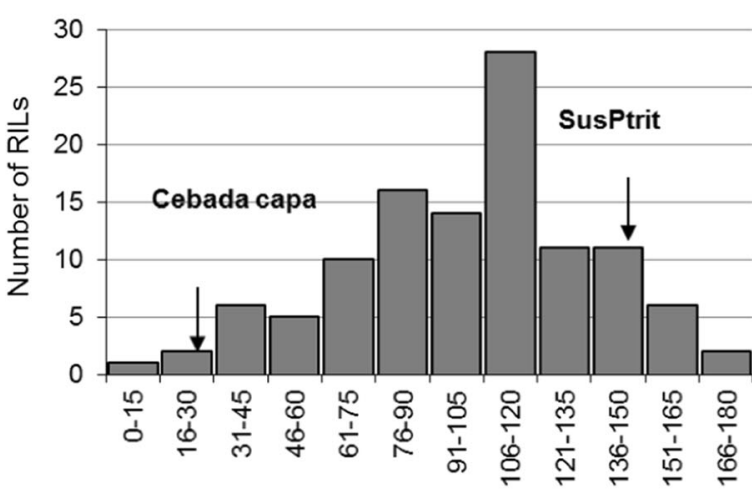

Relative Infection Frequency (RIF)

Fig. 1 Frequency distribution of phenotypes (RLP and RIF) for resistance to Puccinia coronata from Elymus $(P c E)$ in barley mapping populations Vada/SusPtrit and Cebada Capa/SusPtrit. Arrows indicate values of the two parental lines

Table 2 Summary of QTLs conferring resistance to crown rust isolate Puccinia coronata from Elymus repens (PcE) at the seedling stage in two barley mapping populations Vada/SusPtrit (V/S) and Cebada Capa/SusPtrit (CC/S)

\begin{tabular}{|c|c|c|c|c|c|c|c|c|c|}
\hline Population & Trait & QTL & $\mathrm{Chr}$ & $\mathrm{cM}^{\mathrm{a}}$ & Peak marker & $\mathrm{LOD}^{\mathrm{b}}$ & $\%$ Expl & Additive $^{c}$ & Donor $^{\mathrm{d}}$ \\
\hline \multirow[t]{5}{*}{$\mathrm{V} / \mathrm{S}$} & \multirow[t]{3}{*}{ RLP } & $R p c q 1$ & $2 \mathrm{H}$ & 103.3 & E38M54-113 & 5.4 & 10.9 & -1.75 & Vada \\
\hline & & $R p c q 2$ & $5 \mathrm{H}$ & 6.8 & E38M54-247 & 4.8 & 9.9 & 1.70 & SusPtrit \\
\hline & & Rpcq3 & $7 \mathrm{H}$ & 109.9 & E42M55-325 & 5.3 & 10.5 & -1.74 & Vada \\
\hline & \multirow[t]{2}{*}{ RIF } & Rpcq4 & $5 \mathrm{H}$ & 54.8 & E38M54-375 & 4.4 & 10.6 & -8.97 & SusPtrit \\
\hline & & Rpcq3 & $7 \mathrm{H}$ & 95.5 & E35M55-455 & 7.3 & 18.1 & 11.16 & Vada \\
\hline \multirow[t]{5}{*}{$\mathrm{CC} / \mathrm{S}$} & \multirow[t]{3}{*}{ RLP } & $R p c q 5$ & $2 \mathrm{H}$ & 137.3 & E33M61-227 & 7.2 & 20.7 & -3.37 & C. Capa \\
\hline & & Rpcq6 & $3 \mathrm{H}$ & 132.3 & E33M54-356 & 3.6 & 8.6 & -2.25 & C. Capa \\
\hline & & Rpcq7 & $6 \mathrm{H}$ & 147.2 & E42M48-380 & 3.1 & 8.2 & -2.19 & C. Capa \\
\hline & \multirow[t]{2}{*}{ RIF } & $R p c q 7$ & $6 \mathrm{H}$ & 153.8 & E38M61-197 & 3.0 & 9.5 & 10.25 & C. Capa \\
\hline & & $R p c q 8$ & $7 \mathrm{H}$ & 0 & E39M48-310 & 4.0 & 12.8 & -15.36 & SusPtrit \\
\hline
\end{tabular}

QTLs with identical designations are considered the same QTL due to their overlapping in the same chromosomal region $R L P$ relative latency period, $R I F$ relative infection frequency

${ }^{\text {a }}$ Position of the peak marker on the individual linkage maps

b LOD values 3.00 and above were considered QTL

c Effect per copy of the SusPtrit allele on the trait

${ }^{\mathrm{d}}$ Donor of the resistance allele. C. Capa is an abbreviation for Cebada Capa 
irregularity in segregation. The remaining QTLs for resistance were contributed by Vada and Cebada Capa (Table 2; Fig. 2). The support intervals for RLP and RIF overlapped for two of the eight QTLs and, hence, the same gene seems to prolong the LP and to decrease the IF in those to QTLs. These were QTL Rpcq3 on chromosome $7 \mathrm{H}$ in V/S and QTL Rpcq7 on chromosome $6 \mathrm{H}$ in $\mathrm{CC} / \mathrm{S}$ (Table 2).

\section{QTL against $P$. striiformis ff.spp. bromi and tritici}

In V/S, the segregation for number of uredinia and cumulative lesion length was continuous and skewed towards high resistance (Fig. 3). Vada was among the most resistant lines, with SusPtrit among the most susceptible ones for both parameters. Cumulative lesion length and number of uredinia were correlated, with at least $\mathrm{r}=0.9$ for both $P s b$ and Pst.

We detected three QTLs for resistance (Table 3; Fig. 2). Vada contributed all the resistance alleles. For both experiments, and for uredinium number and cumulative lesion length, the mapping resulted in almost identical LOD profiles. Because numbers of uredinia tended to give slightly higher LOD scores than cumulative lesion length, the presented data are for number of uredinia unless indicated otherwise.

The QTL on chromosome $1 \mathrm{H}$ was found in both experiments with $P s b$ and in both experiments with Pst, all with the same peak marker. The QTL on $7 \mathrm{H}$ was less consistent, being discovered only in experiments inoculated with Pst. The number of uredinia of Pst in experiment 2 indicated a QTL with the peak marker at $130.6 \mathrm{cM}$, but the average number of
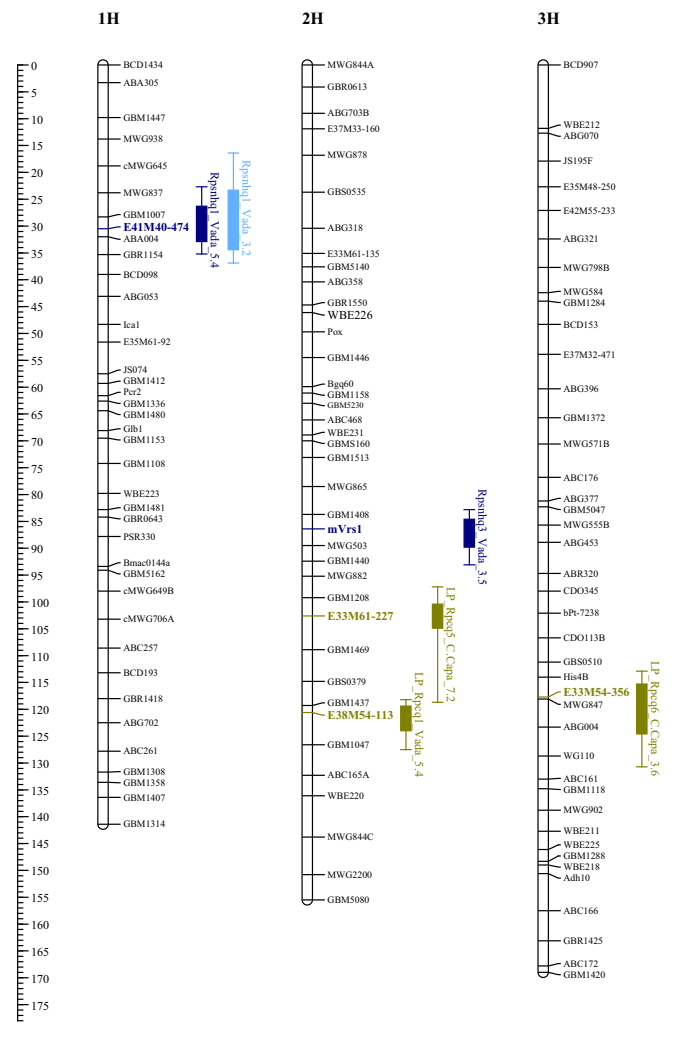

Fig. 2 Integrated linkage map of barley (Aghnoum et al. 2010) showing the QTLs for resistance to three Puccinia striiformis forms [isolates Psh (purple), Pst (light blue) and Psb(dark blue)] and an isolate of $P$. coronata collected from Elymus repens ( $P c E$, olive). QTLs are depicted on the right side of the chromosome bars. The name on QTL bars has three or four components: the provisional name of the gene at the QTL, in some cases preceded by the component of resistance that was
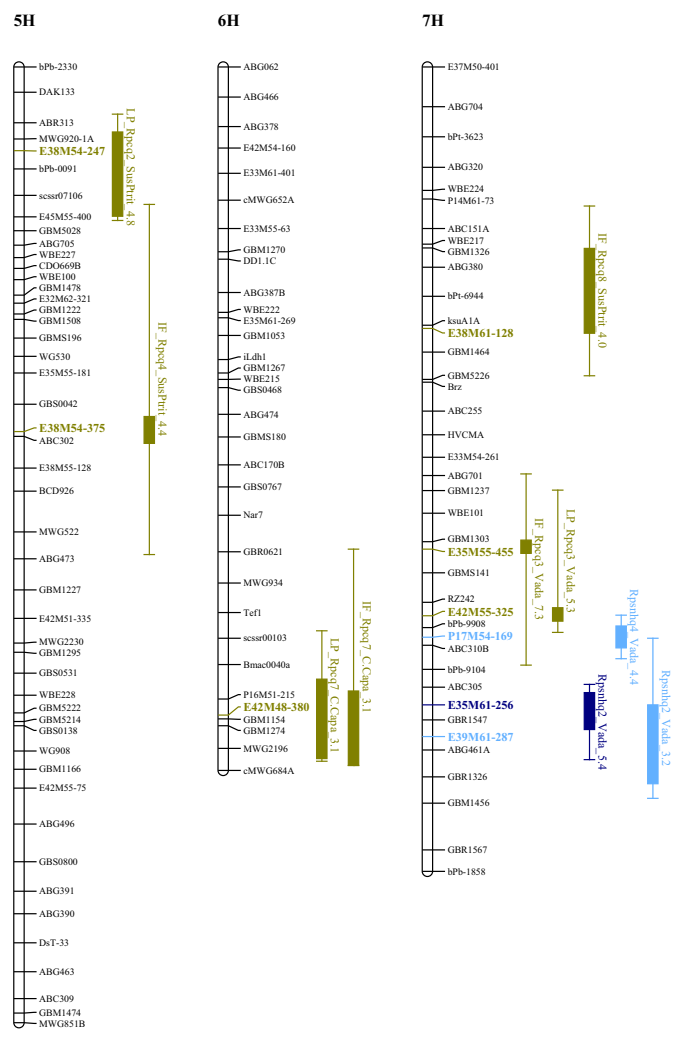

quantified, the cultivar or line that contributed the resistance allele and the LOD value recorded for the QTL. The peak marker of the QTL is highlighted on the chromosome bar by colour and greater font. Similarly coloured QTL bars refer to effectiveness against the same rust isolate. Each QTL bar shows the LOD-1 support interval, the exceeding lines the LOD-2 support interval of each QTL, based on $\mathrm{MMQM}$ results. The ruler on the left side indicates the distances in $\mathrm{cM}$. (Color figure online) 

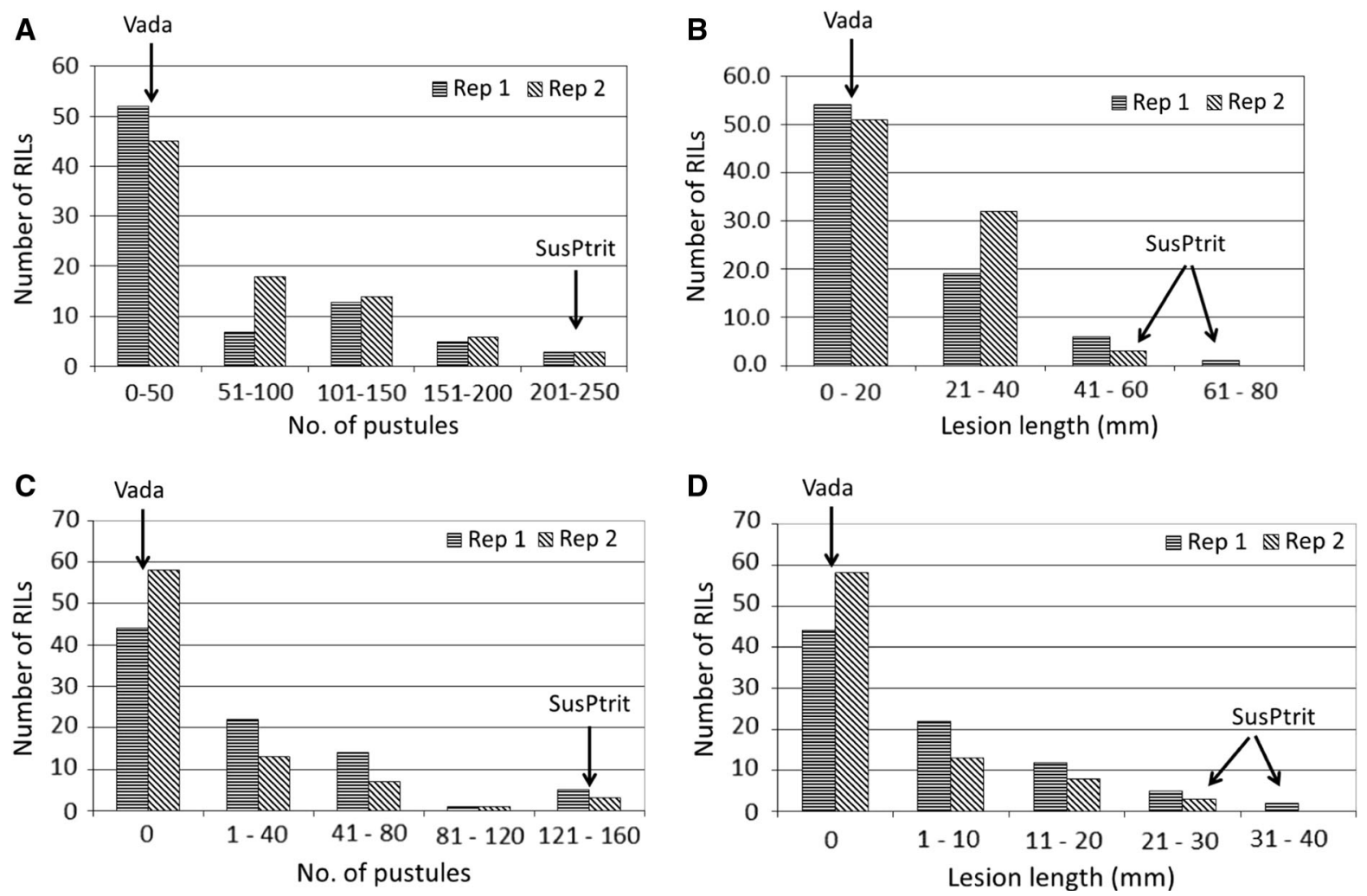

Fig. 3 Frequency distribution of phenotypes (No. of uredinia; Lesion length in $\mathrm{mm}$ ) for resistance to Psb (a, b) and Pst (c, d) in barley mapping population Vada $\times$ SusPtrit for replicates 1 and 2; arrows indicate parental line values

uredinia over experiment 1 and 2 indicated a QTL with the peak marker at $113.7 \mathrm{cM}$. These may or may not refer to the same locus. However, in a settling tower experiment with $P s b$, where uredinia were counted on consecutive days (not published here), a QTL was mapped in the same region (Fig. 2). A third QTL was detected on linkage group $2 \mathrm{H}$; however, only for the average of the data of the two experiments with $P s b$. LOD scores were generally only just significant; for each individual experiment or for the average over the two experiments, LOD score was at most 6 , but more often between 3 and 4 . The percentage of variation explained for individual QTLs ranged from 11 to $26 \%$, but was mostly lower than $20 \%$. This indicates that the (near-)immunity of Vada to $P s b$ and $P s t$ was due to genes with small effects rather than to a major gene for resistance.

\section{QTLs in L/V against $P s h$}

Segregation for number of uredinia and lesion length for L/V with $P s h$ was bimodal, with L94 being among the most resistant and Vada among the most susceptible RILs (Fig. 4). The correlation between number of uredinia and cumulative lesion length was 0.93.

The resistance that segregated in the L/V population mapped to $4 \mathrm{H}$ (Table 3; Fig. 2). The LOD scores were very high at 15-17, and the QTL explained $47 \%$ of the variation observed; hence, this should be considered a major gene for resistance.

Comparison of detected QTLs with QTLs mapped to other rusts in the same mapping populations

In this study, we checked whether the resistance genes contributed by Vada, Cebada Capa, SusPtrit and L94 might also confer resistance to other rust fungi. For this, we compared the positions of the QTLs described above with the positions of QTLs for resistance to rust fungi as reported by Jafary et al. (2008) (Table 4). We considered two QTLs detected for different rusts to co-localise when their LOD-2 support intervals overlapped and they were contributed by the same parental line. Such cases of co-localisation may indicate that the responsible 

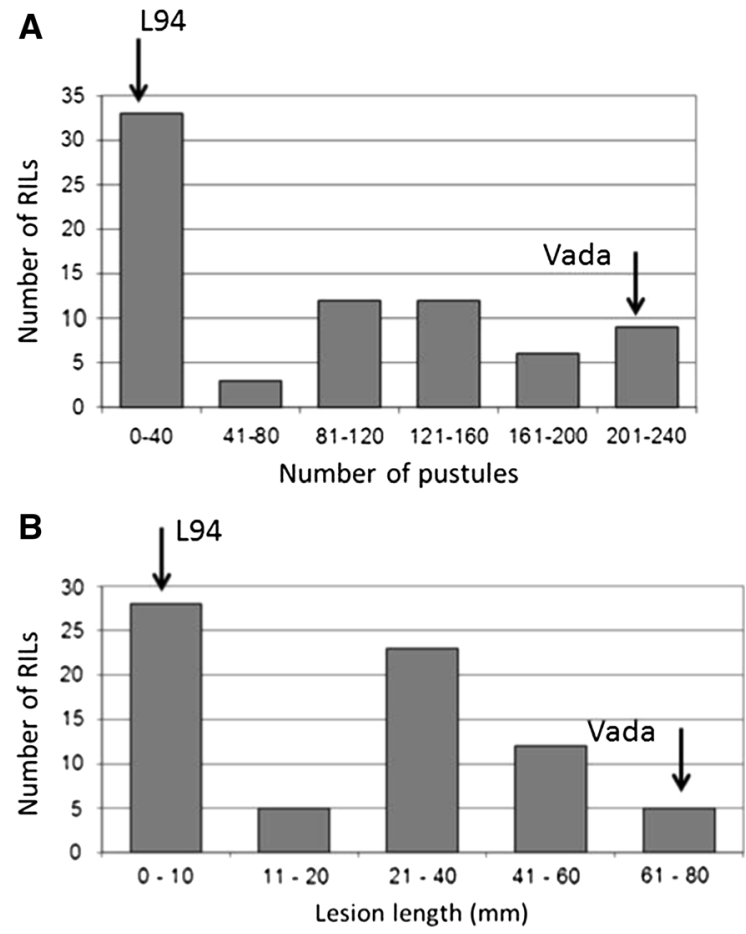

Fig. 4 Frequency distribution of phenotypes (number of uredinia; lesion length in $\mathrm{mm}$ ) for resistance to Psh in barley mapping population L94/Vada; arrows indicate parental line values

gene(s) may be effective against more than one rust species or that they comprise a cluster of quantitative resistance genes, each to a different rust species.

In particular, the resistance QTL to $P c E$, provisionally named Rpcq6 on chromosome $3 \mathrm{H}$ and contributed by 'Cebada Capa', was indicated to localise in a region conferring resistance to four heterologous rust fungi (Table 4; Jafary et al. 2008). QTL Rpcq3 on chromosome 7H also seemed to colocalise with a cluster of resistance genes present in 'Vada' (Table 4; Jafary et al. 2008).

Interestingly, the quantitative resistance gene $R p c$ $q 8$ on $7 \mathrm{H}$, contributed by SusPtrit, co-localised with two QTLs for resistance to two heterologous rusts that were also contributed by the SusPtrit allele. Gene Rpcq4 on $5 \mathrm{H}$ might also be effective against wheat stem rust fungus (Table 4 ).

\section{Discussion}

The mapping populations used in the present study have been used to map resistance to many rust species and isolates, including species that are pathogenic to barley (Puccinia hordei, P. graminis f.sp. tritici) and many species for which barley is a marginal host (Qi et al. 1998; Atienza et al. 2004; Marcel et al. 2007; Jafary et al. 2006, 2008). In the present study, two additional rust species were used to determine the inheritance of resistance. For two of the rust isolates, viz. the P. striiformis of wheat (Pst) and of Bromus $(P s b)$, barley was demonstrated to be a marginal host, and for the other two, the crown rust fungus of $E$. repens $(P c E)$ and the barley yellow rust fungus $(P s h)$, barley was predominantly susceptible at the seedling stage (Niks et al. 2013). The contrasting levels of resistance between the key parental lines of our mapping populations allowed us to determine the genetic basis of the resistance. The levels of resistance of Vada and Cebada Capa against PcE were quantitative. Cebada Capa tended to be relatively resistant as previously described, with Vada appearing nearly as susceptible as SusPtrit. We have no clear explanation for this inconsistency with the results found in our previous study. However, the V/S mapping population segregated for resistance, and QTLs were detected.

We know of only one study on the inheritance of crown rust resistance in barley. Jin and Steffenson (2002) reported that resistance occurred in only approximately $2 \%$ of barley germplasm tested by them and that the resistance in one of the lines was inherited monogenically due to one incompletely dominant gene. That gene, designated Rpcl, mapped to chromosome $3 \mathrm{H}$ in a $30-\mathrm{cM}$ interval containing RAPD and SSR markers (Agrama et al. 2004). Some of those SSR markers were also included in the integrated linkage map used in the present study and were located around the 61- to 64-cM position. None of the resistance loci detected in our study mapped in that region (Fig. 2), and hence, the resistance in our barley lines was not due to Rpcl. In our previous study (Niks et al. 2013), we found a few barley accessions that reacted hypersensitively to $P c E$, and possibly their resistance is due to $R p c 1$. In wheat, a near-nonhost to the barley crown rust, resistance was reported to be due to one (cv Chris) or two (cv Chinese Spring) dominant major genes for resistance (Niu et al. 2014). The resistance found in the present study was quantitative, and we found different QTLs that conferred resistance. The resistance of Vada and Cebada Capa to $P c E$ is partly due to different sets of QTLs: for V/S, on chromosomes $2 \mathrm{H}, 5 \mathrm{H}$ and $7 \mathrm{H}$; for $\mathrm{CC} / \mathrm{S}$, on $2 \mathrm{H}, 3 \mathrm{H}$, 
Table 3 Summary of QTLs conferring resistance of barley seedlings to three isolates of Puccinia striiformis: Psb, collected from Bromus; Pst, collected from wheat; and Psh, representing the barley-infecting form

\begin{tabular}{|c|c|c|c|c|c|c|c|}
\hline$\overline{\text { QTL }}$ & $\mathrm{Chr}$ & $\mathrm{cM}^{\mathrm{a}}$ & Peak marker & Exps with significant QTL $^{\mathrm{b}}$ & $\mathrm{LOD}^{\mathrm{c}}$ & $\%$ Expl & Donor $^{\mathrm{d}}$ \\
\hline \multicolumn{8}{|l|}{$P s b$} \\
\hline Rpsnhq1 & $1 \mathrm{H}$ & 28.1 & E41M40-474 & $1,2,(1+2)$ & 5.4 & 25.1 & Vada \\
\hline Rpsnhq2 & $7 \mathrm{H}$ & 125.8 & E35M61-256 & 3 & 5.4 & 18.6 & Vada \\
\hline Rpsnhq3 & $2 \mathrm{H}$ & 65.2 & mVrs1 & $(1+2)$ & 3.5 & 18.2 & Vada \\
\hline \multicolumn{8}{|l|}{ Pst } \\
\hline Rpsnhq1 & $1 \mathrm{H}$ & 28.1 & E41M40-474 & $1,2^{\mathrm{e}}$ & 3.2 & 11.2 & Vada \\
\hline Rpsnhq2 & $7 \mathrm{H}$ & 130.6 & E39M61-287 & 2 & 3.2 & 13.3 & Vada \\
\hline Rpsnhq4 & $7 \mathrm{H}$ & 113.7 & P17M54-169 & $(1+2)$ & 4.4 & 14.8 & Vada \\
\hline \multicolumn{8}{|l|}{ Psh } \\
\hline Rpsh & $4 \mathrm{H}$ & 72.7 & EBmac0701 & NPUS $^{f}$ & 15 & 46 & L94 \\
\hline Rpsh & $4 \mathrm{H}$ & 75.1 & E40M32-660 & $C L L^{\mathrm{f}}$ & 17 & 49 & L94 \\
\hline
\end{tabular}

Isolates $P s b$ and $P s t$ were tested on mapping population Vada/SusPtrit; isolate $P s h$, on mapping population L94/Vada

a Position of the peak marker on the individual linkage maps

${ }^{\mathrm{b}}$ For experiments 1 and 2, results pertain to the number of uredinia, except when indicated otherwise. $(1+2)$ indicates that the QTL was found on the data averaged over exp 1 and $\exp 2$. Experiment 3 was inoculated in a settling tower, and uredinia were counted on consecutive days (data not presented)

${ }^{c}$ LOD values 3.00 and above were considered QTL

${ }^{\mathrm{d}}$ Donor of the resistance allele

e Only found for cumulative lesion length

${ }^{\mathrm{f}}$ Data for Psh were only from one experiment, and both measurements are presented: number of uredinia (NPUS) and cumulative lesion length (CLL)

$6 \mathrm{H}$ and $7 \mathrm{H}$ (Table 2; Fig. 2). This is similar to the findings for partial resistance to barley leaf rust, caused by P. hordei (Qi et al. 2000; Marcel et al. 2007), and for quantitative resistance to heterologous rusts (Jafary et al. 2008), where barley accessions differed in the location of quantitative resistance genes. One of the QTLs detected in CC/S, Rpcq6 on $3 \mathrm{H}$, may be the broad-spectrum resistance QTL reported by Jafary et al. (2008) to be effective for four heterologous rust species (Table 4). Another QTL, Rpcq8 on 7H, co-localises with a resistance gene contributed by SusPtrit reported by Jafary et al. (2008) and is effective for two heterologous rusts. None of the QTLs detected for resistance to $P c E$ showed a large effect, as the percentage of variance explained was for almost all lower than $15 \%$.

Inheritance of the resistance of barley to $P$. striiformis has been studied previously with the adapted form Psh, the unadapted Pst of wheat (Pahalawatta and Chen 2005; Sui et al. 2010) and the form of barley grass (Golegaonkar et al. 2013), to which barley is a near-nonhost. Inheritance of resistance of barley to the Bromus infecting form of P. striiformis f.sp. bromi (Psb) has, to our knowledge, not been studied before. We found the immunity of Vada to Pst and Psb to be inherited quantitatively. Roughly the same QTLs were detected to both isolates, with the exception of QTL Rpsnhq3 on $2 \mathrm{H}$, which was only found in experiments with $P s b$. This quantitative inheritance contrasts with the monogenic or digenic inheritance of barley resistance to heterologous forms of $P$. striiformis described in previous reports. Golegaonkar et al. (2013) reported that resistance to the barley grass-infecting form was based on one or two major genes. Pahalawatta and Chen (2005) found one dominant major gene (at $4 \mathrm{H}$ ) and one recessive major gene that explained the resistance of barley cultivar Steptoe to Pst. Sui et al. (2010) reported one major gene, on $7 \mathrm{H}$, to be responsible for the resistance in barley line Y12 to an isolate of Pst. These observations contrast with our finding that the immunity of 'Vada' to Pst and Psb was based on a set of genes with relatively small effects. 
Table 4 Summary of QTLs conferring resistance to three isolates of Puccinia striiformis (Psb, from brome grass; Pst, from wheat; and $P$ sh, from barley) and crown rust Puccinia coronata from Elymus repens (PcE) compared with QTLs for nonhost and host resistance mapped to heterologous rusts and two barley-adapted rust fungi ( $P$. hordei and $P$. graminis f.sp. tritici) in the same barley mapping populations, L94/Vada, Vada/SusPtrit and Cebada Capa/SusPtrit, at the seedling stage

\begin{tabular}{|c|c|c|c|c|c|c|c|}
\hline $\mathrm{Chr}$ & Position $(\mathrm{cM})^{\mathrm{a}}$ & Proposed name ${ }^{b}$ & $P s b$ & Pst & $P s h$ & $P c E$ & $\begin{array}{l}\text { Previously mapped QTLs } \\
\text { for resistance to other rust species }\end{array}$ \\
\hline \multirow[t]{2}{*}{$1 \mathrm{H}$} & $16-37$ & Rpsnhq1 & $5.4 ; \mathrm{V}$ & $3.2 ; \mathrm{V}$ & & & $\operatorname{Rphq14}(3 ; \mathrm{V})^{\mathrm{d}}$ \\
\hline & & & & & & & Phm-nhq (4; V) \\
\hline \multirow[t]{3}{*}{$2 \mathrm{H}$} & $83-93$ & Rpsnhq3 & $3.5 ; \mathrm{V}$ & & & & \\
\hline & $118-128$ & Rpcql & & & & $5.4 ; \mathrm{V}$ & $P p-n h q(5 ; \mathrm{V})$ \\
\hline & $97-119$ & $R p c q 5$ & & & & 7.2; CC & \\
\hline \multirow[t]{4}{*}{$3 \mathrm{H}$} & $112-131$ & Rpcq6 & & & & 3.6; CC & Phm-nhq (6; CC) \\
\hline & & & & & & & Phs-nhq (6; CC) \\
\hline & & & & & & & $\boldsymbol{P p}-\boldsymbol{n h} \boldsymbol{q}(7 ; \mathrm{CC})$ \\
\hline & & & & & & & $\boldsymbol{P t}-\boldsymbol{n h} \boldsymbol{q}(8 ; \mathrm{CC})$ \\
\hline $4 \mathrm{H}$ & 104-109 & $R p s h=r p s G Z^{e}$ & & & 16; L94 & & \\
\hline \multirow[t]{2}{*}{$5 \mathrm{H}$} & $9-29$ & $R p c q 2$ & & & & $4.8 ; \mathrm{S}$ & \\
\hline & $26-91$ & Rpcq4 & & & & $4.4 ; S$ & Rpgtq $(5 ; \mathrm{S})$ \\
\hline $6 \mathrm{H}$ & $91-132$ & Rpcq7 & & & & 3.1; CC & \\
\hline \multirow[t]{10}{*}{$7 \mathrm{H}$} & $26-58$ & $R p c q 8$ & & & & $4.0 ; \mathrm{S}$ & $P p-n h q(3 ; S)$ \\
\hline & & & & & & & Phs-nhq (3; S) \\
\hline & $76-111$ & $R p c q 3$ & & & & $7.3 ; \mathrm{V}$ & $P p-n h q(5 ; \mathrm{V})$ \\
\hline & & & & & & & $P t-n h q(11 ; \mathrm{V})$ \\
\hline & & & & & & & $\operatorname{Rphq} 8(3 ; \mathrm{V})$ \\
\hline & & & & & & & $\boldsymbol{P p}-\boldsymbol{n h} \boldsymbol{q}(8 ; \mathrm{V})$ \\
\hline & $102-110$ & Rpsnhq4 & & $4.4 ; \mathrm{V}$ & & & Rpgtq $(5 ; \mathrm{V})$ \\
\hline & & & & & & & $\boldsymbol{P t}$-nhq $(9 ; \mathrm{V})$ \\
\hline & $106-136$ & Rpsnhq2 & $5.4 ; \mathrm{V}$ & $3.2 ; \mathrm{V}$ & & & Phm-nhq (5; V) \\
\hline & & & & & & & $P t-n h q(6 ; \mathrm{V})$ \\
\hline
\end{tabular}

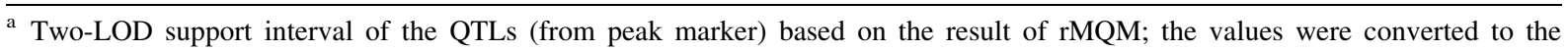
distances on the Barley Integrated Linkage Map (Aghnoum et al. 2010)

b Proposed designation of the QTLs against P. cor. agropyrina (Rpcq), against P. striiformis f.sp. tritici or bromi (Rpsnhq) and against $P$. striiformis f.sp. hordei (Rpsh) discovered in the present study (see Tables 2, 3)

c Data for QTLs effective against other rusts is extracted from Jafary et al. (2008) and Marcel et al. (2007). QTLs are listed for which the resistance allele was donated by the same parent in the same cross and the LOD-2 interval overlapped with the LOD-2 interval of QTLs mapped in the present study. Such QTLs are printed in bold if their peak marker was in the LOD-1 interval of the presently mapped QTLs

Abbreviations for QTLs (-nhq) effective against heterologous rusts: Phm = Puccinia hordei-murini, Phs $=$ P. hordei-secalini, $P p=P$. persistens, $P t=P$. triticina

Abbreviations for QTLs $(R-q)$ to rust fungi that are adapted to barley: ph $=$ Puccinia hordei, pgt $=$ P. graminis f.sp. tritici

${ }^{\mathrm{d}}$ Between parentheses: LOD score; accession contributing the resistance allele: V: Vada; CC: Cebada Capa; S: SusPtrit

e Major gene for resistance reported by Yan and Chen (2006)

The segregation pattern in our mapping population (Fig. 3) would not allow a discrete classification into Resistant: Susceptible on the basis of infection types, as was performed by Golegaonkar et al.
(2013), Pahalawatta and Chen (2005) and Sui et al. (2010). We did not find distinctly different infection types but rather quantitative differences in lesion length and numbers of uredinia. 
The very high resistance of Ethiopian line L94 to $P s h$ was due to a gene with a very large effect located on the long arm of $4 \mathrm{H}$. This gene is most likely the same as that reported by Yan and Chen (2006) to explain the recessive resistance in the Ethiopian line Grannenlose Zweizeilige. That gene, designated rpsGZ, mapped at $7.5 \mathrm{cM}$ from SSR marker EBmac0679, which is included in the integrated linkage map (Aghnoum et al. 2010), and located close to the peak markers for the resistance gene to $P s h$, viz. EBmac0701 and E40M32-660. Because L94 and several other Ethiopian lines were highly resistant not only to Psh but also to Pst and Psb (Niks et al. 2013), this gene may be universally effective against $P$. striiformis. The functional dominant allele RpsGZ may be a susceptibility gene, as discussed by Pavan et al. (2010), similar to the well-known Mlo gene of barley to powdery mildew. Such genes may be broad spectrum and durably effective (Pavan et al. 2010). If the resistance gene on $4 \mathrm{H}$ in $\mathrm{L} 94$ is rpsGZ, this gene may also be effective against other isolates and formae speciales of $P$. striiformis. It will, however, not be effective against other rust species because L94 is extremely susceptible to $P$. hordei and unusually susceptible to several heterologous grass and cereal rusts, including $P$. triticina and $P$. hordei-murini (Atienza et al. 2004). Thus, if the gene represents a susceptibility gene, it is apparently rust species specific.

The present study illustrates again that immune responses may be based on the aggregate effect of minor genes for resistance. The absence of major genes in the interactions between barley and Pst and $P s b$ seems to be at odds with the hypothesis by Schulze-Lefert and Panstruga (2011) that nonhost resistance against pathogens of plants that are closely related to the nonhost are mostly based on major $R$ genes. The co-incidence of LOD-1 support intervals of several QTLs for resistance to $P c E$, with peak markers for QTLs to heterologous rusts, suggests that such genes may have broad effectiveness against several rust species or occur as clusters of closely linked, rustspecific genes.

Acknowledgments We are grateful to the Netherland Fellowship Program (NUFIC) for financing SKA. TCM was supported by the BIOEXPLOIT Integrated Project that resides under the 6th framework programme of the European Union. Our gratitude also extends to Anton Vels for the technical support and to Dr. Ana M. González for her advice in mapping the QTLs. The Ethiopian Institute of Agricultural Research (EIAR) is also acknowledged for facilitating SKA to participate in this study.

\section{Compliance with Ethical Standards}

Conflict of interest The authors declare that they have no conflict of interest.

Open Access This article is distributed under the terms of the Creative Commons Attribution 4.0 International License (http:// creativecommons.org/licenses/by/4.0/), which permits unrestricted use, distribution, and reproduction in any medium, provided you give appropriate credit to the original author(s) and the source, provide a link to the Creative Commons license, and indicate if changes were made.

\section{References}

Aghnoum R, Niks RE (2010) Specificity and levels of non-host resistance of barley to non-adapted Blumeria graminis forms. New Phytol 185:275-284

Aghnoum R, Marcel TC, Johrde A, Pecchioni N, Schweizer P, Niks RE (2010) Basal resistance of barley to barley powdery mildew: connecting QTLs and candidate genes. Mol Plant Microbe Interact 23:91-102

Agrama HA, Dahleen L, Wentz M, Jin Y, Steffenson B (2004) Molecular mapping of the crown rust resistance gene Rpc1 in barley. Phytopathology 94:858-861

Atienza SG, Jafary H, Niks RE (2004) Accumulation of genes for susceptibility to rust fungi for which barley is nearly a nonhost results in two barley lines with extreme multiple susceptibility. Planta 220:71-79

Chen WQ, Wellings C, Chen XM, Kang ZS, Lu TG (2014) Wheat stripe (yellow)rust caused by Puccinia striiformis f.sp. tritici. Mol Plant Pathol 15:433-446

Collins NC, Niks RE, Schulze-Lefert P (2007) Resistance to cereal rusts at the plant cell wall-what can we learn from other host-pathogen systems? Aust J Agric Res 58:476-489

Golegaonkar PG, Wellings CR, Singh D, Park RF (2013) Genetic and molecular analyses of resistance to a variant of Puccinia striiformis in barley. J Appl Genet 54:1-9

Jafary H, Szabo LJ, Niks RE (2006) Innate nonhost immunity in barley to different heterologous rust fungi is controlled by sets of resistance genes with different and overlapping specificities. Mol Plant Microbe Interact 19:1270-1279

Jafary H, Albertazzi G, Marcel TC, Niks RE (2008) High diversity of genes for nonhost resistance of barley to heterologous rust fungi. Genetics 178:2327-2339

Jansen RC, Stam P (1994) High resolution of quantitative traits into multiple loci via interval mapping. Genetics 136:1447-1455

Jin Y, Steffenson BJ (2002) Sources and genetics of crown rust resistance in barley. Phytopathology 92:1064-1067

Jørgensen JH (1992) Discovery, characterization and exploitation of Mlo powdery mildew resistance genes in barley. Euphytica 63:141-152 
Marcel TC, Varshney RK, Barbieri M, Jafary H, de Kock MJD, Graner A, Niks RE (2007) A high-density consensus map of barley to compare the distribution of QTLs for partial resistance to Puccinia hordei and of defence gene homologues. Theor Appl Genet 114:487-500

Niks RE (1987) Nonhost plant species as donors for resistance to pathogens with narrow host range: I. Determination of nonhost status. Euphytica 36:841-852

Niks RE (2014) How specific is non-hypersensitive host and nonhost resistance of barley to rust and mildew fungi? J Integr Agric 13:244-254

Niks RE, Marcel TC (2009) Nonhost and basal resistance: how to explain specificity? New Phytol 182:817-828

Niks RE, Walther U, Jaiser U, Martínez F, Rubiales D, Andersen O, Flath K, Gymer P, Heinrichs F, Jonsson R, Kuntze L, Rasmussen M, Richter E (2000) Resistance against barley leaf rust (Puccinia hordei) in West-European spring barley germplasm. Agronomie 20:769-782

Niks RE, Parlevliet JE, Lindhout P, Bai Y (2011) Breeding crops with resistance to diseases and pests. Wageningen Academic Publishers, Wageningen

Niks RE, van Heyzen S, Szabo LJ, Alemu SK (2013) Host status of barley to Puccinia coronata from couch grass and $P$. striiformis from wheat and brome. Eur J Plant Pathol 136:393-405

Niu ZX, Puri KD, Chao SM, Jin Y, Sun YL, Steffenson BJ, Maan SS, Xu SS, Zhong SB (2014) Genetic analysis and molecular mapping of crown rust resistance in common wheat. Theor Appl Genet 127:609-619

Pahalawatta V, Chen X (2005) Genetic analysis and molecular mapping of wheat genes conferring resistance to the wheat stripe rust and barley stripe rust pathogens. Phytopathology 95:427-432
Pavan S, Jacobsen E, Visser RGF, Bai Y (2010) Loss of susceptibility as a novel breeding strategy for durable and broad-spectrum resistance. Mol Breed 25:1-12

Qi X, Niks RE, Stam P, Lindhout P (1998) Identification of QTLs for partial resistance to leaf rust (Puccinia hordei) in barley. Theor Appl Genet 96:1205-1215

Qi X, Fufa F, Niks RE, Lindhout P, Stam P (2000) The evidence for abundance of QTLs for partial resistance to Puccinia hordei on the barley genome. Mol Breed 6:1-9

Schulze-Lefert P, Panstruga R (2011) A molecular evolutionary concept connecting nonhost resistance, pathogen host range, and pathogen speciation. Trends Plant Sci 16:117-125

Sui X, He Z, Lu Y, Wang Z, Xia X (2010) Molecular mapping of a non-host resistance gene YrpstY1 in barley (Hordeum vulgare L.) for resistance to wheat stripe rust. Hereditas 147:176-182

Van Ooijen JW (1992) Accuracy of mapping quantitative trait loci in autogamous species. Theor Appl Genet 84:803-811

Van Ooijen JW (2004) MapQTL ${ }^{\circledR} 5$, software for the mapping of quantitative trait loci in experimental populations. Kyazma B.V., Wageningen

Voorrips RE (2002) MapChart: software for the graphical presentation of linkage maps and QTLs. J Hered 93:77-78

Yan GP, Chen XM (2006) Molecular mapping of a recessive gene for resistance to stripe rust in barley. Theor Appl Genet 113:529-537

Yeo FKS, Hensel G, Vozábová T, Martin-Sanz A, Marcel TC, Kumlehn J, Niks RE (2014) Golden SusPtrit—a genetically well transformable barley line for studies on the resistance to rust fungi. Theor Appl Genet 127:325-337 